\section{A una década del año del pulmón}

\section{A decade of the year of the lung}

\section{José Luis Sandoval-Gutiérrez*}

*Instituto Nacional de Enfermedades Respiratorias Ismael Cosío Villegas. Ciudad de México, México.

Citar como: Sandoval-Gutiérrez JL. A una década del año del pulmón. Neumol Cir Torax. 2021; 80 (3): 236. https://dx.doi.org/10.35366/102495

Me gusta vivir, respirar mejor que trabajar... mi arte es el de vivir. Marcel Duchamp

El año 2010 fue nombrado por el FIRS (Forum of International of Respiratory Societies) ${ }^{1}$ «el año del pulmón», esto debido a que, un año previo, la pandemia de influenza A H1N1 se había presentado. ${ }^{2-5}$

Es importante resaltar que los objetivos de esa nominación contemplaban:

1. Promover a nivel global la atención de la salud pulmonar y sus enfermedades.

2. Incrementar el financiamiento a la investigación del área respiratoria.

3. Desarrollar e implementar herramientas para prevenir las enfermedades pulmonares y discapacidad de las mismas.

4. Diagnosticar y tratar las neumopatías en el curso inicial de la enfermedad.
5. Investigar nuevos métodos y medicamentos para el tratamiento de las enfermedades torácicas.

6. Impulsar programas para promover la salud respiratoria.

Existen avances en cada uno de los puntos, pero aún queda mucho trabajo por hacer. Sigue pendiente la formación de una Federación de Tórax, ${ }^{6}$ y la promoción de la Neumología y Cirugía de Tórax en los diferentes sectores de la salud pública ya que las neumopatías son la primera causa de mortalidad en el mundo, ${ }^{7}$ pero han sido infraestimadas $y$ hasta ignoradas por quienes toman decisiones gubernamentales.

Hay que enfatizar que las enfermedades respiratorias no sólo son un problema de salud, sino también económico, ya que la enfermedad pulmonar obstructiva crónica (EPOC) es en la actualidad la tercera causa de muerte en el mundo, las neumonías la cuarta y la tuberculosis la décima. ${ }^{8}$ Existe un problema importante con el asma, la adicción al tabaco y el cáncer con más letalidad que es el pulmonar. Todos ellos generan discapacidad y pérdida de años de vida.

El advenimiento del COVID-19 nos mostró lo vulnerable que está el orbe ante una crisis respiratoria. Debemos seguir trabajando en promover y difundir, no sólo en estudiar, trabajar e investigar sobre los tópicos pulmonares.

Debemos influir en nombrar la «década del pulmón» 2020-2030, ya que un año no fue suficiente, es necesario para los pacientes y los profesionales que nos dedicamos a este campo del saber, el beneficio es mutuo y necesario.

\section{REFERENCIAS}

1. Forum of International Respiratory Societies. Access date: 2020 Jun 16. Available from: https:// www.firsnet.org/

2. Guntupalli KK, Gutterman D, Raoof S, Markowski PA. 2010: The year of the lung. Chest. 2010;138(6):1287-1288. Available from: https:// doi.org/10.1378/chest.10-2555

3. Siafakas NM, Decramer M, Rabe KF. 2010 the year of the lung. Eur Respir J. 2011;37(1):1-2. Available from: https://doi.org/10.1183/09031936.00171210

4. Siafakas N. The year of the lung 2010. Pneumon. 2010;23(4):336-337.

5. ALAT. 2010: Año del pulmón. [Fecha de consulta 17 de junio de 2020] Disponible en: https:// alatorax.org/es/novedades/2010-ano-delpulmon

6. Sandoval-Gutiérrez JL. Hacia una federación mundial de tórax. Nuestro reto. Neumol Cir Torax. 2008;67(4):161.

7. Sandoval-Gutierrez JL, Reyes ES, Bautista EB. Pulmonary diseases: first cause of mortality in the world. Chest. 2011;139(6):1550. Available from: https://doi.org/10.1378/chest.11-0021

8. Top ten causes of death. [Access date 2020 Jun 18] Available from: https://www.who.int/ gho/mortality_burden_disease/causes_death/ top_10/en/

Conflicto de intereses: El autor declara no tener conflicto de intereses.

Correspondencia:

Dr. José Luis Sandoval-Gutiérrez Instituto Nacional de Enfermedades

Respiratorias Ismael Cosío

Villegas, Ciudad de México.

Correo electrónico:

sandovalgutierrez@gmail.com 